\title{
Route Optimization using Forecasting, Wheelchair Modelling and Path Planning
}

\author{
David Sanders, \\ Giles Tewkesbury, \\ Malik Haddad, MIEEE, \\ Zoubir Zouaoui, \\ Sarinova Simandjuntak
}

\begin{abstract}
In routing systems, the capability to generate reliable safe routes depends on the integration of a path optimization algorithm with dynamics and models. In this work, a system based on a novel route optimization algorithm is considered. The sensitivity of the lowest energy solutions is analyzed considering different simplified wheelchair models. The aim is to implement a system for routing wheelchairs around obstacles so that more people can safely drive powered wheelchairs. Powered wheelchairs can positively impact participation, activity, wellbeing, pain and self-esteem. Systems described here can allowing more autonomous or collaborative driving, and enhance mobility of disabled individuals finding it difficult to use standard powered wheelchairs. The work included a small qualitative exploratory study that exposed three topics: (a) "situations that might benefit from path planning and obstacle avoidance; (b) "optimism concerning the use of technological systems on powered wheelchairs"; (c) "characteristics of disabled users that could benefit from using a powered wheelchair". Findings indicated that the systems presented in this paper could help to overcome some of the difficulties in using powered wheelchairs.
\end{abstract}

\section{INTRODUCTION}

This work describes an innovative decision support system for powered wheelchairs. The system delivers innovative routing for obstacle avoidance. Route selection is optimized by attempting to find the route that uses the least energy consumption but that is safe.

Real-time data from sensors is exploited through data assimilation algorithms [1]. The ability of obstacle avoidance algorithms to generate reliable optimal routes critically depends on the interplay of the optimization and the wheelchair model. In this work the sensitivity of the optimized routes is investigated. A novel route optimization algorithm was used. Results of the first numerical tests of the system are described. The tests were performed to examine the effect, on the optimized routes, of different approaches to the computation of components. Sensitivity analysis was conducted by considering a wheelchair moving through an environment containing some obstacles. The different least energy solutions obtained by the optimization algorithm were comparatively analyzed.

The World Health Organization estimated that 65 million people worldwide need wheelchairs [2]. The 2010 United States Census reported 3.6 million wheelchair users aged over fifteen [3] and in the USA in 2002 , there were 2,700,000 non-institutionalized users of wheeled mobility devices, approximately $1,800,000$ of which used powered wheelchairs or scooters [4]. Europe has reported similar data [5,6]. The use of powered wheelchairs will probably continue to increase as the number of disabled people worldwide increases as demographics change and populations age with a corresponding rise in persistent health 
conditions [7]. It is therefore beneficial to make sure that powered wheelchairs best meet user needs to help with their involvement and improve their quality of life.

Advantages of self-mobility using powered wheelchairs include enhanced self esteem [8], pain reduction [9], and increases in social participation and activity [10-13]. But using a powered wheelchair is difficult for some disabled people, Drivers have reported being scared to drive within crowded spaces [14] and clinicians have reported that various people cannot use powered wheelchairs safely because of cognitive, motor and visual deficits [15-17]. Smart powered wheelchairs can provide support and can be useful for disabled people who have difficulty using, or cannot use a wheelchair at all. Many of the wheelchair systems are not available outside of research laboratories and have never moved beyond the development phase but wheelchairs developed at the University of Portsmouth are successfully in use and the research in this paper is proposed for them. The wheelchairs are providing assistance to people with severe cognitive, sensory or motor limitations [17-20].

\section{ROUTE OPTIMIZATION}

The three main blocks of the routing system were:

- Forecasting

- Wheelchair modelling

- Route optimization.

\section{A. Forecasting}

The forecasting chain used for the numerical tests described in this paper used models of the wheelchair and the environment. The approach adopted to develop the forecasting component was based on gradually upgrading a baseline structure. Steps were:

a) Dynamic modelling component, for forecasting reaction to obstacles and veer due to surface changes $[21,22]$.

b) Data assimilation system to ingest data collected from the user joystick and ultrasonic sensors [23-28].

c) Prediction System based on systems being developed for routing ships [29,30] to estimate reliability of optimized routing solutions.

Forecast and route optimization reliability measures were key elements in the decision making process, especially in complicated environments.

\section{B. Wheelchair Performance Modelling}

Forecasts of wheelchair performance could be generated using dynamics models. These were included in path optimization algorithms to find optimal routes around obstacles, accounting for optimization instances (save energy, reduce danger, and / or minimize transit time), and the constraints (the obstacles). A good "working compromise" was found between computational times (affecting system response times) and modelling details (affecting quality and reliability of routing). A primary characteristic was the computational optimization of the used software that obtained "best results in the least time". Effort was devoted to this goal.

The overall computational load of the algorithms was large. To speed-up wheelchair performance computation, some computing was completed off-line and results stored in Look-Up Tables (LUT) for wheelchair response and performance. In the first implementations, the computation of such LUTs was based on simple approaches and the numerical tests served to benchmark and improve the algorithmic structure. Sensitivity analysis was conducted for the route optimization algorithm (described below). Wheelchair modelling details were added gradually in the off-line wheelchair modelling component (in order to improve wheelchair data) and coded in the LUTs. In the future, the format of the LUTs will be gradually standardized.

In the first computing tests, energy consumption minimization for the wheelchair was considered, using the decomposition of a reduced set of the main components described by Lewis in [23]. Total energy, $\mathrm{W}_{\text {tot, }}$ was made up from three components:

$$
\mathrm{W}_{\text {tot }}=\mathrm{W}_{\text {dynamics }}+\mathrm{W}_{\text {obstacle }}+\mathrm{W}_{\text {veer }}
$$


Where $\mathrm{W}_{\text {dynamics }}$ was the dynamics model of the wheelchair, and was tabulated as a function of wheelchair speed across flat ground and included as part of the wheelchair specific LUTs.

$\mathrm{W}_{\text {obstacle }}$ was the obstacle added resistance using the potential field-based method where an artificial repulsive potential field repelled the wheelchair from obstacles. It was evaluated as:

$$
\mathrm{W}_{\text {obstacle }}=\mathrm{k} f_{\text {rep }}
$$

Where $\mathrm{k}$ was a constant and the potential field calculations for $f_{\text {rep }}$ were:

$$
f_{\text {rep }}= \begin{cases}-k_{\text {rep }} \sum_{i=1}^{n}\left(\frac{1}{d_{i}}-\frac{1}{d_{\text {max }}}\right) s_{i}, & \text { if } d_{i}<d_{\max } \\ 0, & \text { othewise }\end{cases}
$$

Where $s_{i .}=\left(\mathrm{r}-\mathbf{o}_{\mathbf{i}}\right) /\left|\mathrm{r}-\mathbf{o}_{\mathbf{i}}\right|$

Equation (3) is the calculation for the repulsive field that pushed the wheelchair away from obstacles in which $\mathbf{O}_{\mathbf{i}}$ was the position vector of an obstacle. Fig. 1 shows the repulsive field. The straight (purple) line is the path of the wheelchair with the joystick pushed forward. The number and length of the curved (blue) lines show the strength of the repulsive force.

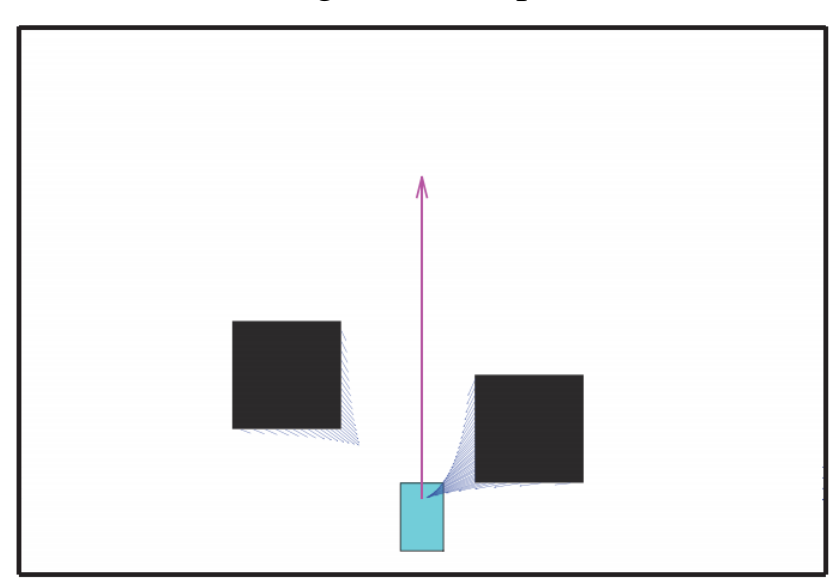

Figure 1. Repulsive field (from [24].

After receiving distance data from the range sensor(s) then objects within a threshold range (for example 2.5 meters) are considered. They were enlarged with regard to the wheelchair size and a Gaussian (repulsive) potential field was constructed from it. Fig. 2 is the structural diagram of the process.

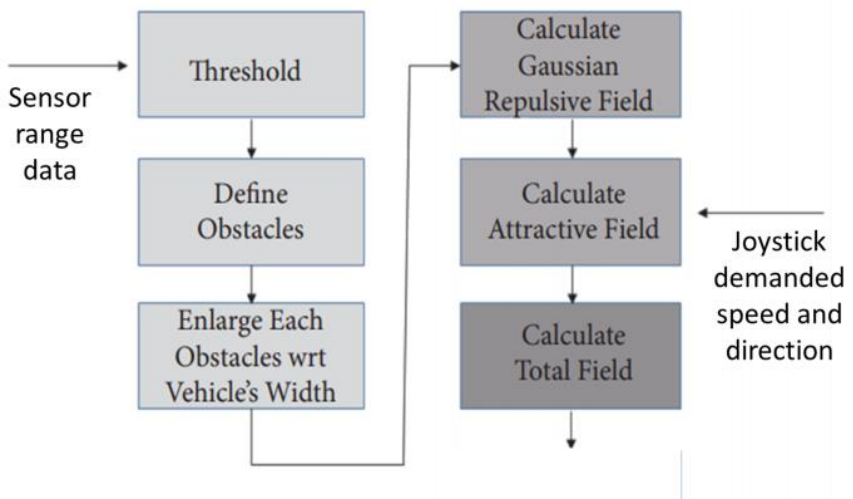

Figure 2. Structural diagram for creating a repulsive field around obstacles.

Details of $\mathrm{W}_{\text {obstacle }}$ could be accounted for by wheelchair specific coefficients and corresponding joystick terms.

$\mathrm{W}_{\text {veer }}$ was the added factor due to sloping ground, and was evaluated as:

$$
\mathrm{W}_{\text {veer }}=\mathrm{H}_{\mathrm{s}} \mathrm{C}_{\text {slope }}
$$

Where $\mathrm{H}_{\mathrm{s}}$ was the slope and the coefficient $\mathrm{C}_{\text {slope }}$ embodied the interaction between the wheelchair and the slope. In the first implementation of the system a simple structure was assumed for it, i.e. only the change 
in wheelchair direction. In further developments of the system, speed and direction will be included. The dependence on these parameters could be stored in the LUTs as specific wheelchair responses.

\section{Optimization}

Route optimization algorithms used a new approach to the routing problem. Avoiding discretization of the research domain into a graph, the algorithm explored the domain and produced solutions for every given point from a single origin (point of departure) or target destination (point of arrival).

The algorithm was designed to resolve the general minimum cost path searching problem in a continuous domain and for nonstationary conditions. Different approaches to the combined effect of obstacle and slopes on the results could be adopted and integrated within the route optimization algorithm.

\section{TESTING}

The first computational tests of the system used a dataset corresponding to a Bobcat II wheelchair passing between two obstacles as shown in Fig. 1. A simplified approach was adopted with several (numerically) interchangeable variants defined by considering different parametrizations of the coefficients. The goal of this model was to test the effects of several simple, but physically plausible, combinations of obstacles and wheelchair movements dictated by a joystick. Other data was taken from the literature.

Two variants for angular dependency were defined to correspond to the interaction of the wheelchair with surfaces characterized as smooth or rough, or sloping or flat. A linear dependence on wheelchair speed was assumed to account for the variability of values that can be found by different approaches available in the literature. Speed and turn (velocity) provided measures of energy consumption and performance as determined from wheelchair dimensions and technical tests and data in manuals. In a more generalized optimal routing philosophy, besides route shape (time and speed profile), optimization was included by computing energy consumption for different configurations.

A set of wheelchair routes was selected and several drives were simulated going from a start point to a target destination. An example is shown in Fig. 3.

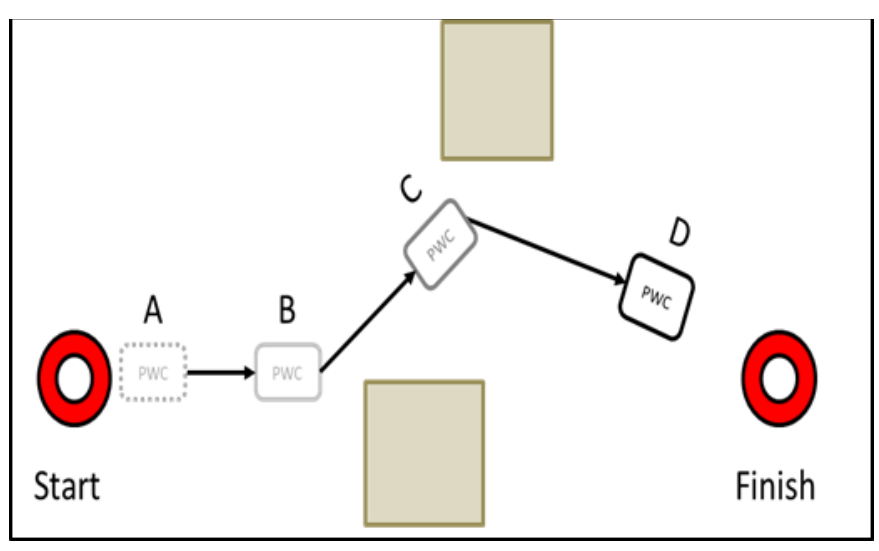

Figure 3. A wheelchair test route with two offset obstacles.

A constant wheelchair speed and turning rate were assumed. Differences in wheelchair speed produced different energy consumption rates. Solutions were the geometrically shortest routes. Total energy consumption for all the optimized routes was calculated and all the paths produced by the system successfully avoided obstacles.

\section{QUALITATIVE STUDY}

An exploratory study was undertaken with some structured interviews to obtain perspectives regarding the use of the new methods. The study was run as part of a group project during an extended undergraduate degree at the University of Portsmouth.

Individual interviews were conducted. Before each interview, information about powered wheelchair use and some socio-demographic information was gathered by means of a socio-demographic questionnaire. That individual data (for example sex and age) and data about wheelchair use (for example control methods and how often they have used a powered wheelchair) were documented. A semi-structured interview guide with probes and open-ended questions was used. That was adapted during the interviews to catch any themes that emerged during the interview. Volunteers were asked about current and past use of wheelchairs, 
including aspects that were largely positive (for example, benefits or activities performed with their wheelchair) as well as aspects that were more challenging (for example, safety, accidents, barriers, needs). After that, the volunteers were shown a short film showing the new functionality available on the Smart Wheelchair. The film facilitated some informed discussion about relevance and demonstrated primary characteristics of the new systems created by the research team. Volunteers were asked questions about their perception of the new systems after the film (for example, relevance, confidence, safety and use), and some more specific questions about relevance (e.g., obstacle avoidance, path following, etc.). Carers were asked about their role and what they perceived to be the potential impact of the new work.

Preliminary analysis took place while data were being collected. Each interview was firstly scrutinized for any general thoughts. Once analyzed individually then each interview was subjected to more in-depth analysis to identify any predominant themes. Differences in opinion were settled during discussion amongst project team members.

The wheelchair was controlled by a joystick and had ultrasonic sensors mounted on it. It could help a user to safely follow a path, avoid obstacles and negotiate doorways and gaps.

When considering current wheelchair use and potential use of the new systems, three main themes emerged:

(a) Challenges may be overcome by the new systems;

(b) Cautious optimism concerning the new systems;

(c) Personalizing the systems to each wheelchair user.

\section{A. Challenges may be Overcome by the new Systems}

Users described the "freedom", "independence" and "autonomy" that their wheelchair gave to them. Although, wheelchair users and caregivers recounted many challenging situations that had occurred. These most commonly related to:

- Indoor Environment: impenetrable buildings, small elevators, tight entry doorways, store aisles, narrow spaces created by temporary set ups and / or displays.

- Outdoors: rain and snow, pavements in poor condition, poor visibility, long distances.

- Crowded places: shopping centers, festivals, stadiums.

People described strategies they had adopted to overcome many of their challenging situations and avoid difficult situations. They found alternative locations or activities, or relied on caregivers or others for assistance. Some strategies could compromise safety, for example using bicycle or bus lanes instead of pavements. People described incidents and accidents, ranging from banging into doorframes or shop fittings and scraping knuckles to being hit by a bus or car.

After viewing the video showing the new systems, volunteers discussed whether the new features could alleviate some of their challenges. Obstacle avoidance was especially popular and identified as something that could reduce frequently encountered difficulties, for example exiting narrow or crowded elevators, or driving through crowded places without hitting other people.

\section{B. Cautious Optimism Concerning the new Systems}

Volunteers were enthusiastic about what they saw but did express concern, regarding their ability and about using or understanding the new technology. They wanted to continue doing things they could physically do and did not want the wheelchair to replace their own abilities. Volunteers mostly only perceived some features as being relevant. Several were skeptical about trusting the new wheelchair systems more than their own abilities or instinct, or were concerned about reliability. Others thought the opposite and felt the systems would respond more quickly in emergencies.

Some people were concerned about technical characteristics, for example how would sensors be fitted to their chair and would they increase chair width. Making a chair wider would make it more difficult to drive, especially in narrow spaces or doorways. People were also concerned about outdoor activities and whether their modified chair would be too slow. People did not like the fact that the systems could not detect pavement edges, holes, cracks, or items on the floor. People were especially worried about the new systems not identifying red traffic lights. Because the new systems were still being developed, people provided 
some useful and interesting technical suggestions, for example "include an auditory signal when approaching obstacles".

\section{Personalizing the Systems to each Wheelchair User}

People found at least two features could be helpful: obstacle avoidance and path following. They felt the new systems could be especially useful when they were tired. The new features could reduce cognitive and physical demands, thereby increasing independence and reducing the need for carers. Volunteers identified some characteristics of people likely to benefit, for example: tiredness, reduced vision, slow reactions and poor upper extremity motor control. It was also suggested that the new systems will become more useful as people age, and the number of visual and cognitive impairments increased. The systems could be more reassuring for elderly people. Overall, the new systems were considered useful for people with decreased autonomy.

The new systems could be more beneficial as medical condition deteriorated (for example multiple sclerosis), in particular as upper extremity control was reduced or lost, or as fatigue increased. The systems could be useful to people with slow progressive spinal muscular atrophy as their condition could deteriorate to the point where hand motor control was lost.

\section{CONCLUSIONS}

A system based on wheelchair performance models with a route optimization algorithm was successfully implemented. Results from initial tests demonstrated that satisfactory and safe routes were selected. Tests were performed to benchmark the algorithms, and to produce optimized routes using different simplified approaches to computation.

Results from the routing solutions were satisfactory, and a main point that emerged was that although obstacles might add time and distance to routes and the geometrical shapes of the routes varied considerably, variations in total route length were relatively small (variations were up to only about $2.5 \%$ ).

Routes with obstacles were completed more quickly and safely (without any collisions) with the new systems assisting drivers. Usability, efficacy and safety were satisfactory. The wheelchair avoided obstacles and collisions and the drivers were able to navigate routes.

Results from the qualitative study indicated that people often encountered challenges with their wheelchairs. People thought there were benefits to the new systems, but they did raise some important concerns and they had some questions. Results provided information for further development of the systems. To date, most studies had focused on testing and developing Smart features, for example, Nguyen et al. [31] described a wheelchair with a brain-computer interface.

When people were questioned about whether they would use the new systems, half the volunteers said that they would use it now and $75 \%$ said they might use it in the future. Some said they might feel safer or that pedestrians might feel safer. Those who did not want to use the new system said they were less relevant to them because they could drive well at the moment, but they identified others who could benefit.

People in the study felt that obstacle avoidance and path following could help to overcome some reported challenges. Some people said that if the new systems were available now they would not choose to use them. Some felt they had better driving abilities than the new automated systems. And some were concerned that the systems may make the wheelchair too slow. That was similarly reported in two studies concerning the perception of older inhabitants of long-term care homes [32,33]. These results emphasized the significance of understanding and accounting for the needs of wheelchair drivers.

A significant challenge was designing technology for a range of abilities [34]. Although many people thought at least two of the new functions were useful in this study, several people thought at least one function would not be useful for them. Knowing some but not all features might be appropriate, refinement of the systems could allow users to select options appropriate to them.

\section{FUTURE WORK}

Volunteers gave their feedback after watching a film showing the new systems rather than from personal experience. Their perspective might have differed if they had experienced the new systems. Future work will test the systems during clinical trials at Chailey Heritage Foundation.

The perspective of clinicians would also be valuable to know and future studies could include caregivers. People in this study did not reflect the health conditions, diversity and age range of the general population 
using powered wheelchairs. Additionally, the volunteers did not include anyone with a cognitive deficit even though some of the new systems were aimed at that population. Findings cannot be generalized to other settings.

An aspect of validity is the data credibility $[35,36]$. The perspective of the interviewer and the possible impact of that perspective on the data needs to be considered in the future.

Results did suggest that the new systems had potential to improve participation. Some people said that driving a wheelchair fitted with the new systems could change their experience of going shopping etc. Further analysis is needed to understand how the new systems might impact social participation. Additionally, future work will include new measurement methods and tools to assess wheelchair users use in various natural settings [37].

Further work is investigating improving wheelchair steering [38-41].

\section{REFERENCES}

[1] E. Kalnay. Atmospheric Modeling, Data Assimilation and Predictability, Cambridge Univ. Press, p.341, 2003.

[2] World Health Organization. Guidelines on the Provision of Manual Wheelchairs in Less Resourced Settings; WHO Press: Geneva, Swizerland, 2008.

[3] USA Census Bureau. U.S. Disability Statistics and Information. Available online: http://www.disabled-world.com/disability/statistics/info.php (accessed 20 Feb 2021).

[4] J. Flagg. Wheeled Mobility Demographics. In Industry Profile on Wheeled Mobility; Rehabilitation Engineering Research Center on Technology Transfer: University at Buffalo, Buffalo, NY, USA, 2009.

[5] B. Sapey, J. Stewart, and G. Donaldson. The Social Implications of Increases in Wheelchair Use; Lancaster University: Lancaster, UK, 2004.

[6] A. Van Drongelen, B. Roszek, E. Hilbers-Modderman, M. Kallewaard, C. Wassenaar. Wheelchair Incidents; National Institute for Public Health and the Environment (RIVM): Bilthoven, Netherlands, 2002.

[7] World Health Organization. World Report on Disability 2011; WHO: Geneva, Switzerland, 2011.

[8] I. Pettersson, G. Ahlström, K. Törnquist. The value of an outdoor powered wheelchair with regard to the quality of life of persons with stroke: A followup study. Assist. Technol. 2007, 19, 143-153.

[9] A. Davies, L. de Souza, and A. Frank. Changes in the quality of life in severely disabled people following provision of powered indoor/outdoor chairs. Disabil. Rehabil. 2003, 25, 286-290.

[10] A. Frank, J. Ward, N. Orwell, C. McCullagh, M. Belcher. Introduction of a new nhs electric powered indoor/outdoor chair (Epioc) service: Benefits, risks and implications for prescribers. Clin. Rehabil. 2000, 14, 665-673.

[11] A. Brandt, S. Iwarsson, and A. Stahle. Older people's use of powered wheelchairs for activity and participation. J. Rehabil. Med. 2004, 36, 70-77.

[12] A. Salminen, A. Brandt, K. Samuelsson, O. Toytari, A. Malmivaara. Mobility devices to promote activity and participation: A systematic review. J. Rehabil. Med. 2009, 41, 697-706.

[13] C. Löfqvist, C. Pettersson, S. Iwarsson, and A. Brandt. Mobility and mobility-related participation outcomes of powered wheelchair and scooter interventions after 4-months and 1-year use. Disabil. Rehabil. 2012, 7, 211-218.

[14] S. Evans, A. Frank, C. Neophytou, L. de Souza. Older adults' use of, and satisfaction with, electric powered indoor/outdoor wheelchairs. Age Ageing 2007, 36, 431-435.

[15] L. Fehr, W. Langbein, S. Skaar. Adequacy of power wheelchair control interfaces for persons with severe disabilities: A clinical survey. J. Rehabil. Res. Dev. 2000, 37, 353-360.

[16] W. Mortenson, L. Clarke, and K. Best. Prescribers' experiences with powered mobility prescription among older adults. Amer. J. Occup. Ther. 2013, $67,100-107$.

[17] D. Sanders, M. Haddad, P. Omoarebun, F. Ikwan, J. Chiverton, S. Zhou, I. Rogers, \& B. Vatchova. Intelligent control and HCI for a powered wheelchair using a simple expert system and ultrasonic sensors. Intelligent Systems and Applications Vol 3. Advances in Intelligent Systems \& Computing, vol. 1252, Springer, pp. 571-583, 2020.

[18] D. Sanders, A. Gegov, M. Haddad, F. Ikwan, D. Wiltshire, and Y. Tan, "A rule-based expert system to decide on direction and speed of a powered wheelchair,". Proc. of SAI Intelligent Systems Conference, London, pp. 822-838. 2018.

[19] M. Haddad, D. Sanders, M. Langner, P. Omoarebun, M. Thabet, and A. Gegov, "Initial results from using an intelligent system to analyse powered wheelchair users' data," Proc. 2020 IEEE 10th International Conference on Intelligent Systems (IS), Varna, 2020, pp. 241-245.

[20] M. Haddad, D. Sanders, M. Langner, F. Ikwan, G. Tewkesbury, and A. Gegov, "Steering direction for a powered-wheelchair using the Analytical Hierarchy Process,". In Proc. 2020 IEEE 10th International Conference on Intelligent Systems (IS), Varna, pp. 229-234. 2020.

[21] D. Sanders, M. Langner, N. Bausch, Y. Huang, S. Khaustov, \& S. Simandjuntak. Improving human-machine interaction for a powered wheelchair driver by using variable-switches and sensors that reduce wheelchair-veer. Intelligent Syst \& Apps. Advances in Intelligent Systems \& Computing, vol. 1038, Springer, pp. 1173-1191, 2019.

[22] D. Sanders, M. Langner, G. Tewkesbury, "Improving wheelchair-driving using a sensor system to control wheelchair-veer and variable-switches as an alternative to digital-switches or joysticks," Ind Rob: An int' jnl., vol. 32, no. 2, pp.157-167, 2010.

[23] M. Haddad, G. Tewkesbury, M. Thabet, P. Omoarebun, \& T. Barker. Simple expert system for intelligent control and HCI for a wheelchair fitted with ultrasonic sensors. IEEE Proc' of 10th Int Conf on

[24] D. Sanders, G. Tewkesbury, H. Parchizadeh, J. Robertson, P. Omoarebun, \& M. Malik, Learning to drive with and without intelligent computer systems and sensors to assist. Intelligent Systems and Applications Volume 1. Advances in Intelligent Systems and Computing, vol. 868, Springer, pp. 1171$1181,2018$.

[25] Sanders, D, Gegov, A, Tewkesbury, G \& Khusainov, R 2019, Sharing driving between a vehicle driver and a sensor system using trust-factors to set control gains. Intelligent Systems and Applications Volume 1. Advances in Intelligent Systems and Computing, vol. 868, Springer, pp. 1182-1195, 2018.

[26] D. Sanders, B. Sanders, A. Gegov \& D. Ndzi. Results from investigating powered wheelchair users learning to drive with varying levels of sensor support. Intelligent Systems Conference (IntelliSys). IEEE, pp. 241-245, 2017.

[27] D. Sanders, B. Sanders, A. Gegov \& D. Ndzi. Using confidence factors to share control between a mobile robot tele-operater and ultrasonic sensors. Intelligent Systems Conference (IntelliSys). pp. 1026-1033, 2017.

[28] D. Sanders, D. Ndzi, S. Chester, \& M. Malik. Adjustment of tele-operator learning when provided with different levels of sensor support while driving mobile robots. Lecture Notes in Networks and Systems, vol. 16, Springer, United Kingdom, pp. 548-558, 2016.

[29] M. Hoffschildt,J. Bidlot, B. Hansen, P. Janssen,. Potential benefit of ensemble forecasts for ship routing, ECMWF Research Department Tech. Memo. 287, ECMWF, Reading. 1999.

[30] C. Jang-Ho, P. Dong-Sung, L. Myo-Taeg, and K. Tae-Koo. A Real-Time Obstacle Avoidance Method for Autonomous Vehicles Using an ObstacleDependent Gaussian Potential Field. Journal of Advanced Transportation, Vol 2018, pp 1-16. 2018. 
[31] J. Nguyen, S. Su, H. Nguyen. Experimental Study on a Smart Wheelchair System Using a Combination of Stereoscopic and Spherical Vision. Proceedings of Annual International Conference of the IEEE Engineering in Medicine and Biology Society, Osaka, Japan, 3-7 July 2013; Volume 2013, pp. 4597-4600.

[32] P. Viswanathan, J. Little, A. Mackworth, A. Mihailidis. Navigation and Obstacle Avoidance Help (Noah) for Older Adults with Cognitive Impairment: A Pilot Study. In Proceedings of ACM SIGACCESS Conference on Computers and Accessibility (ASSETS), Dundee, Scotland, 24-26 October 2011; pp. 43-50.

[33] R. Wang, A. Mihailidis, T. Dutta, G. Fernie. Usability testing of multimodal feedback interface and simulated collision-avoidance power wheelchair for long-term-care home residents with cognitive impairments. J. Rehabil Res. Dev. 2011, 48, 801-822.

[34] Y. Choi, S. Sprigle. Approaches for evaluating the usability of assistive technology product prototypes. Assist. Technol. $2011,23,36-41$.

[35] E. Guba, Y. Lincoln, Fourth Generation Evaluation; Sage Publications: Newbury Park, CA, USA, 1989; p. 294. [Google Scholar]

[36] A. Shenton. Strategies for ensuring trustworthiness in qualitative research projects. Educ. Inform. 2004, $22,63-75$.

[37] M. Haddad, D. Sanders, M. Langner, M. Thabet, P. Omoarebun, A. Gegov, N. Bausch, and K. Giasin, "Intelligent system to analyze data about powered wheelchair drivers," Proc. of SAI Intelligent Systems Conference, Amsterdam, 2020, pp. 584-593.

[38] D. Sanders, and N. Bausch, "Improving steering of a powered wheelchair using an expert system to interpret hand tremor," in Proc. International Conference on Intelligent Robotics and Applications, Portsmouth, pp. 460-471. 2015.

[39] M. J. Haddad, and D. A. Sanders, "Deep Learning architecture to assist with steering a powered wheelchair," IEEE Trans. Neur. Sys. Reh., vol. 28, no 12, pp. 2987-2994, 2020.

[40] M. J. Haddad, and D. A. Sanders, "Selecting a best compromise direction for a powered wheelchair using PROMETHEE," IEEE Trans. Neur. Sys. Rehab., vol. 27, no. 2, pp.228-235, 2019.

[41] M. Haddad, D. Sanders, A. Gegov, M. Hassan, Y. Huang, and M. Al-Mosawi, "Combining multiple criteria decision making with vector manipulation to decide on the direction for a powered wheelchair," in Proc. SAI Intelligent Systems Conf, London, pp. 680-693. 2019. 